\title{
Epithelial TRAF6 drives IL-17-mediated psoriatic inflammation
}

\author{
Reiko Matsumoto, ${ }^{1}$ Teruki Dainichi, ${ }^{1}$ Soken Tsuchiya, ${ }^{2}$ Takashi Nomura, ${ }^{1}$ Akihiko Kitoh, ${ }^{1}$ \\ Matthew S. Hayden, ${ }^{3}$ Ken J. Ishii, ${ }^{4,5}$ Mayuri Tanaka, ${ }^{4,5}$ Tetsuya Honda, ${ }^{1}$ Gyohei Egawa, ${ }^{1}$ \\ Atsushi Otsuka, ${ }^{1}$ Saeko Nakajima, ${ }^{1}$ Kenji Sakurai, ${ }^{1}$ Yuri Nakano, ${ }^{1}$ Takashi Kobayashi, ${ }^{6}$ \\ Yukihiko Sugimoto, ${ }^{2}$ and Kenji Kabashima ${ }^{1,7}$
}

'Department of Dermatology, Kyoto University Graduate School of Medicine, Kyoto, Japan. ²Department of Pharmaceutical Biochemistry, Kumamoto University Faculty of Life Sciences, Kumamoto, Japan. ${ }^{3}$ Section of Dermatology, Department of Surgery, Dartmouth-Hitchcock Medical Center, Lebanon, New Hampshire, USA. ${ }^{4}$ Laboratory of Adjuvant Innovation, National Institutes of Biomedical Innovation, Health and Nutrition, Osaka, Japan. ${ }^{5}$ Laboratory of Vaccine Science, WPI Immunology Frontier Research Center, Osaka University, Osaka, Japan. ${ }^{6}$ Department of Infectious Disease Control, Faculty of Medicine, Oita University, Oita, Japan. ${ }^{7}$ Singapore Immunology Network (SIgN) and Institute of Medical Biology, Agency for Science, Technology and Research (A*STAR), Biopolis, Singapore.

Epithelial cells are the first line of defense against external dangers, and contribute to induction of adaptive immunity including Th17 responses. However, it is unclear whether specific epithelial signaling pathways are essential for the development of robust IL-17-mediated immune responses. In mice, the development of psoriatic inflammation induced by imiquimod required keratinocyte TRAF6. Conditional deletion of TRAF6 in keratinocytes abrogated dendritic cell activation, IL-23 production, and IL-17 production by $\gamma \delta$ T cells at the imiquimod-treated sites. In contrast, hapteninduced contact hypersensitivity and papain-induced IgE production were not affected by loss of TRAF6. Loss of psoriatic inflammation was not solely due to defective imiquimod sensing, as subcutaneous administration of IL-23 restored IL-17 production but did not reconstitute psoriatic pathology in the mutant animals. Thus, TRAF6 was required for the full development of IL-17mediated inflammation. Therefore, epithelial TRAF6 signaling plays an essential role in both triggering and propagating IL-17-mediated psoriatic inflammation.

Conflict of interest: The authors have declared that no conflict of interest exists.

Submitted: March 19, 2018 Accepted: June 21, 2018 Published: August 9, 2018

\section{Reference information:} JCI Insight. 2018;3(15):e121175 https://doi.org/10.1172/jici. insight.121175.

\section{Introduction}

Epithelial tissues provide a physical barrier between the host and the external environment. Epithelial cells are capable of sensing pathogens and environmental factors and produce immunomodulatory factors including a variety of cytokines and chemokines (1). It is now well accepted that epithelial products influence the nature of the innate and adaptive immune responses (2). For example, epithelial cells promote $\mathrm{T}$ helper 2-type (Th2-type) immune responses by releasing interleukin 33 (IL-33), thymic stromal lymphopoietin (TSLP), and IL-25 in allergic airway inflammation (3) and atopic dermatitis (4). Similarly, epithelial cells secrete serum amyloid A (SAA) in response to segmented filamentous bacteria (SFB) colonization, promoting Th17-type immune responses in murine intestine (5). Conditional deletion of genes encoding immunomodulatory factors specifically in epithelial cells has established that epithelial cells do shape the immune response in vivo (6-10). However, it is less clear how specific epithelial signaling events are mechanistically linked to qualitative changes in the nature of the immune response (11-13).

Psoriasis is a common, chronic skin disease with histological features of abnormal proliferation and differentiation of keratinocytes accompanied with massive infiltration of immune cells $(14,15)$. First, environmental factors, such as physical trauma and bacterial products, initiate a cascade of events at the epidermis in genetically predisposed individuals. Second, continuous activation of keratinocytes by IL-17 family cytokines leads to disease maintenance and propagation. Production of IL-17 is driven by the IL-23/IL-17 axis in which dendritic cells (DCs) produce IL-23, which supports Th17-dependent or -independent production of IL-17 in the skin $(16,17)$. Keratinocytes release multiple factors that contribute to both phases of psoriasis pathogenesis. In response to tissue damage, keratinocytes release the cellular damage-associated molecular patterns (DAMPs) including self-nucleic acids and high-mobility group box 1 protein (HMGB1). 
Keratinocytes also produce inducible proinflammatory transcriptional products, such as chemokine (C-C motif) ligand 20 (CCL20) and chemokine (C-X-C motif) ligand 1 (CXCL1) (15), that act on the immune cells that induce psoriatic inflammation. Consequently, it is suggested that the interplay of keratinocytes and immune cells orchestrates an IL-17-dependent, feed-forward inflammatory loop in psoriasis. However, it is not known whether keratinocytes, or a specific signaling pathway within keratinocytes, are truly required for initiating the IL-23/IL-17 axis in skin.

Tumor necrosis factor (TNF) receptor-associated factor 6 (TRAF6) is a signaling adaptor and E3 ubiquitin ligase that can regulate the activation of nuclear factor- $\mathrm{KB}(\mathrm{NF}-\kappa \mathrm{B})$ and mitogen-activated protein kinase (MAPK) pathways. TRAF6 plays an important role in a variety of immunological functions through the regulation of MAPK and NF- $\kappa B$ activation $(18,19)$. Genetic alterations that affect the NF- $\mathrm{kB}$ and MAPK pathways in the epidermis have been associated with genetic predisposition to psoriasis (20-22) and suggest a possible role for TRAF6 in the disease. Specifically, gain-of-function mutations in caspase recruitment domain-containing protein 14 (CARD14), which is predominantly expressed in the epidermis and signals through TRAF6, are related to a familial type of psoriasis $(23,24)$. Likewise, in animals, the psoriatic phenotype is induced by keratinocyte-specific gene deletion of Tnip1 coding A20 binding and inhibitor of NF- $\mathrm{kB}-1$ (ABIN-1) (12). ABIN-1, which binds to polyubiquitin chains and interacts with the deubiquitinase A20, restricts activation of NF- $\mathrm{KB}$ and MAPK signaling by inhibiting polyubiquitination of TRAF6 (25). In addition, IL-17-mediated psoriatic dermatitis is induced by topical application of imiquimod (IMQ), a ligand for Toll-like receptor 7/8 (TLR7/8), which signals through TRAF6 (26). Based on these studies, which suggest a role for TRAF6 in psoriasis pathogenesis, we set out to use conditional gene deletion to directly assess the contribution of keratinocyte TRAF6 in murine models of cutaneous inflammation.

Here, we show that keratinocyte-specific TRAF6-deficient mice are fully resistant to IMQ-induced psoriatic inflammation. In the absence of epithelial TRAF6, IMQ fails to induce activation of either skinresident DCs or $\gamma \delta$ T cells. This abrogation of psoriatic dermatitis in mice lacking epithelial TRAF6 results from loss of 2 signaling events. First, TRAF6 in keratinocytes regulates the primary response to external stimuli, and second, TRAF6 is required for the positive feedback loop through the IL-17 receptor (IL-17R). Thus, these findings conclusively demonstrated that TRAF6 signaling in keratinocytes is critical for the establishment of IL-17-dependent psoriatic inflammation in the skin.

\section{Results}

TRAF6 in keratinocytes is essential for the development of IMQ-induced psoriatic dermatitis. To address the role of TRAF6-dependent signaling in epithelial cells in cutaneous inflammation, we generated keratinocyte-specific TRAF6-deficient (K5-Cre; Traf6 $\left.{ }^{f / f f}\right)$ mice, hereafter referred to as Traf6 epithelial knockout (Traf6EKO) mice, by crossing keratin 5-Cre (K5-Cre) transgenic mice with mice carrying floxed Traf6 alleles (Traf6 ${ }^{f / f t}$; Supplemental Figure 1A; supplemental material available online with this article; https://doi.org/10.1172/ jci.insight.121175DS1).

To evaluate the role of epidermal TRAF6 in IL-17-mediated inflammation, we used a well-established model, IMQ-induced dermatitis, which is characterized by an IL-23/IL-17 axis-dependent psoriatic inflammation (16). Consistent with previous reports, repetitive topical application of IMQ to the ears of Traf $6^{f / f l}$ mice induced skin inflammation with marked erythema, scaling, and crust (Figure 1A). In contrast, no obvious inflammation was observed in Traf6EKO mice treated with IMQ. Histological examination revealed that IMQ-treated skin of Traf6 ${ }^{f / f l}$ mice was characterized by hyperplasia of the epidermis, with massive infiltration of inflammatory cells, recapitulating human psoriatic dermatitis (Figure 1B). On the other hand, Traf6EKO mice failed to exhibit these histological changes following IMQ treatment (Figure 1B). The increased ear thickness following IMQ application on WT mice was abrogated in Traf6EKO mice during the observation period (Figure 1C). Flow cytometric analysis of the lesional skin infiltrates revealed a profound increase of total living cells and $\mathrm{CD} 45^{+}$cells, including neutrophils and $\mathrm{CD} 4^{+} \mathrm{T}$ cells, in Traf6 $6^{\text {Alfl }}$ mice but not in Traf6EKO mice (Figure 1, D and E). There was also a striking reduction in infiltrating neutrophils, which are characteristic of psoriatic inflammation in this animal model (27), in Traf6EKO mice compared with Traf6 $6^{f l f l}$ mice following IMQ treatment (Figure 1D). V $\gamma 4^{+} \gamma \delta$ T cells are the dominant source of IL-17 in IMQ-induced inflammation (28). We observed reduced $\mathrm{V} \gamma 4^{+} \gamma \delta \mathrm{T}$ cells and IL-17 production by these cells was significantly decreased in Traf6EKO mice compared with Traf6 $6^{\text {flfl }}$ mice following IMQ treatment (Figure 1, D-F). These results demonstrated that loss of TRAF6 in epithelial cells renders mice resistant to IMQ-induced psoriatic inflammation. 
A

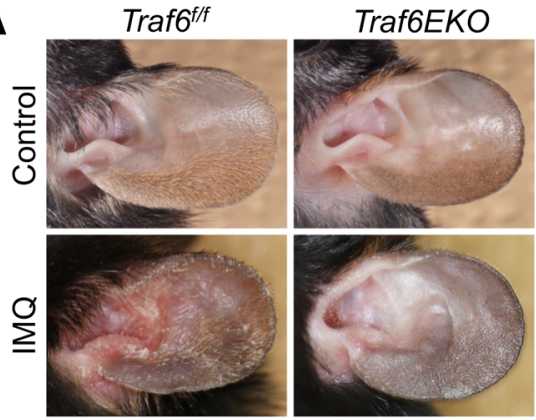

C

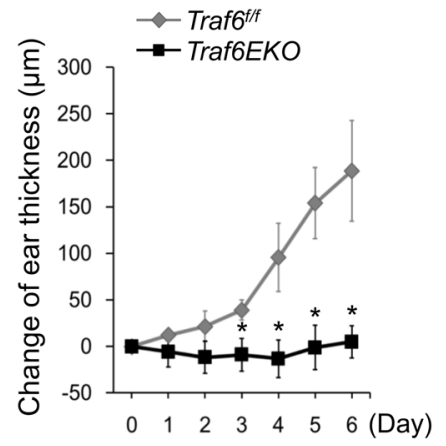

E

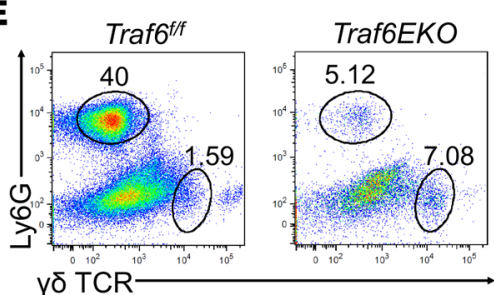

F

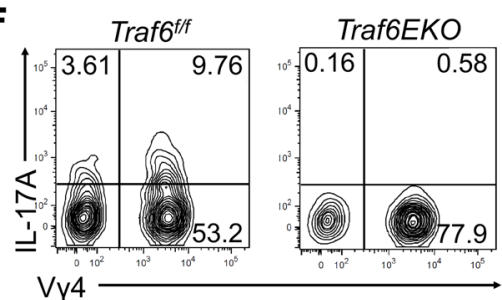

B

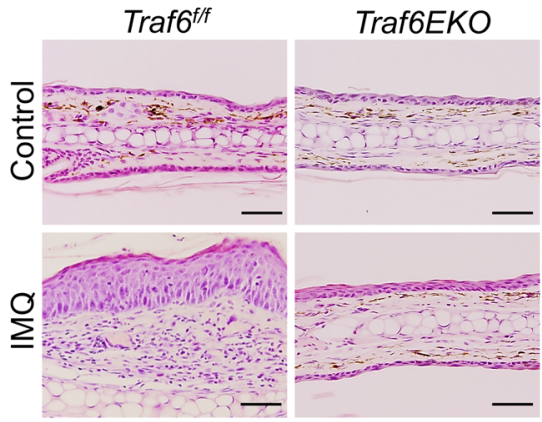

D

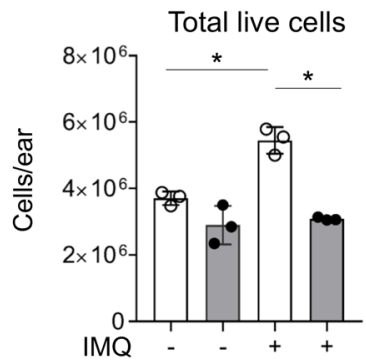

$\square$ Traf6ff

Traf6EKO
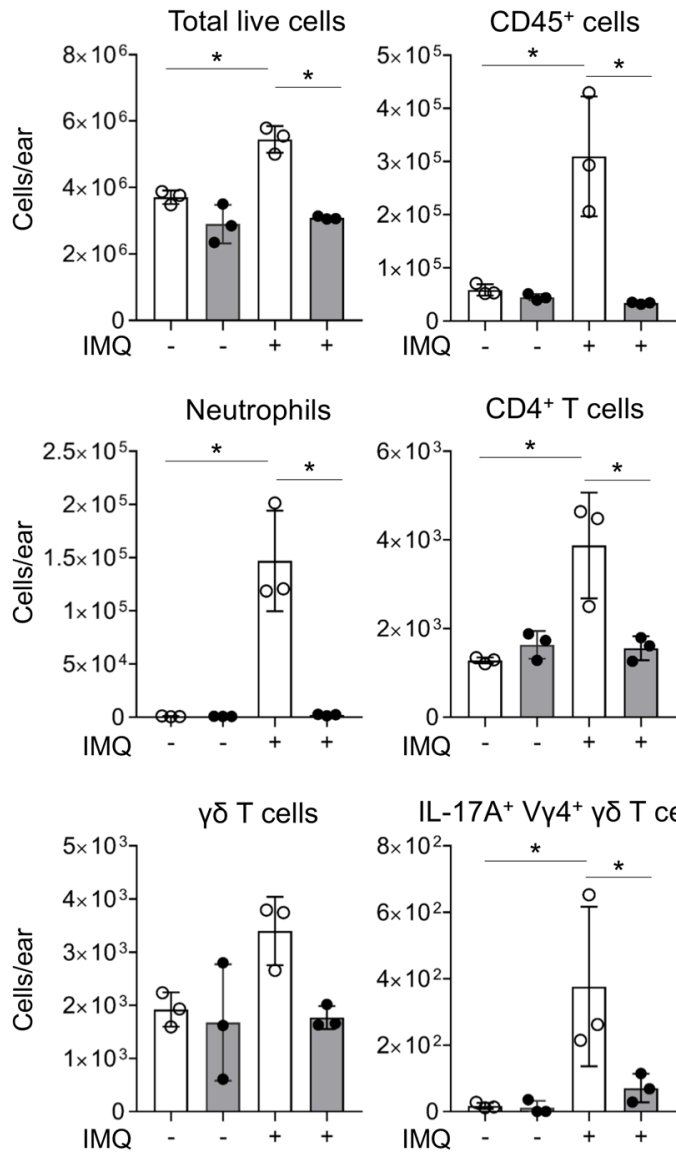

Figure 1. Traf6EKO mice are resistant to psoriatic inflammation induced by IMQ. (A) Representative gross appearance of ears from Traff $f^{f / f l}$ and Traf6EKO mice treated daily with or without topical IMQ for 6 consecutive days. Traf6EKO mice showed no remarkable gross abnormality in the skin except for modest hair reduction in the auricles and postauricular regions. (B) Histology of the ear sections harvested on day 6. H\&E staining. Scale bars: $50 \mu \mathrm{m}$. (C) Time course of changes in the ear thickness ( $n \geq 4$ per group). The thickness was measured daily before treatment. Values are shown as means \pm SD. ${ }^{*} P<0.05$ (2-tailed Student's $t$ test). (D) Flow cytometric analyses of total live cells, CD45+ cells, CD4+ $4^{+}$cells, Ly6C ${ }^{+}$neutrophils, $\gamma \delta$ TCR $^{+}$cells, and $\gamma \delta$ TCR $^{+} V \gamma 4^{+} I L-17 A^{+}$ cells from the ears of individual mice after 3 days of IMQ treatment $(n=3)$. Results are expressed as means \pm SD. ${ }^{*} P<0.05$ (Tukey's multiple-comparisons test). (E and F) Representative flow cytometry plots of neutrophils and IL-17A-producing $\gamma \delta$ T cells infiltrated in the ear of Traf6 $6^{f / f l}$ and Traf6EKO mice treated with IMQ on day $3(n=3)$. Dot plots show Ly6 $\mathrm{C}^{+}$neutrophils $(\mathbf{E})$ gated on CD45+ cells and $\mathrm{V} \gamma 4^{+} \mathrm{IL}-17 \mathrm{~A}^{+}$cells $(\mathbf{F})$ gated on $\gamma \delta$ TCR ${ }^{\text {int }}$ cells shown in $\mathbf{E}$. Data are representative of at least 3 independent experiments $(\mathbf{A}-\mathbf{C})$ or 2 experiments $(\mathbf{D}-\mathbf{F})$.

Traf6EKO mice may exhibit reduced expression of Traf6 in thymic epithelial cells, as these cells are also known to express keratin 5 (29). Furthermore, mice with Traf6 deletion in Foxn1-expressing thymic epithelial cells are reported to have abnormalities in the negative selection of $\mathrm{T}$ cells, leading to abnormal $\mathrm{T}$ cell populations including $\mathrm{CD} 4^{+} \mathrm{CD} 8^{+}$double-positive populations of $\mathrm{T}$ cells in their peripheral tissues (30). 
Flow cytometric analysis showed that the absolute numbers of $\mathrm{CD}^{+} \mathrm{T}$ cells, $\mathrm{CD} 8^{+} \mathrm{T}$ cells, and $\mathrm{CD} 4^{+} \mathrm{CD} 8^{+}$ $\mathrm{T}$ cells in the thymus and lymph nodes were similar between Traf6EKO and Traf6 $6^{A / f}$ mice (Supplemental Figure 1B). The numbers of $\mathrm{CD}^{+} \mathrm{T}$ cells and $\gamma \delta \mathrm{T}$ cells in the skin of Traf6EKO mice were higher than those of $T r a f 6^{f / f l}$ mice, whereas those of $\mathrm{V} 4^{+} \gamma \delta \mathrm{T}$ cells in the skin of both groups were comparable (Supplemental Figure 1B). Nevertheless, despite potentially autoreactive $\mathrm{T}$ cells in the TrafbEKO skin related to the expected reduction of TRAF6 expression in the thymus (30), there were no obvious signs of cutaneous inflammation (Figure 1, A and B).

Langerhans cells (LCs) are the dominant professional antigen-presenting cells in the epidermis, and our previous work has suggested that LCs produce IL-23 and induce IL-17-producing cells in the IMQ-induced murine psoriatic dermatitis model (31). In contrast to Traf6EKO mice, IMQ treatment of Langerin-Cre; Traf6 $^{\text {flfl }}$ (Traf6LCKO) mice lacking TRAF6 specifically in LCs induced robust inflammation similar to that observed in Traf6 ${ }^{\sharp / f l}$ mice (Supplemental Figure 1, C and D). These findings indicated that TRAF6-dependent signaling in keratinocytes, not in LCs, is required for the development of psoriatic inflammation.

IL-17-mediated immunity induced by IMQ depends on TRAF6 in keratinocytes. To more broadly characterize the impact of TRAF6 in keratinocytes on IMQ-induced psoriatic inflammation, we analyzed gene expression in IMQ-treated whole ears of Traf6EKO mice and Traf6 ${ }^{l / f l}$ mice using a cDNA microarray. At day 2, prior to the prominent skin immune infiltrate evident on day 3 (Figure 1, B-D), increased expression of several proinflammatory genes was detectable. As shown in the heatmap, 406 genes were upregulated more than 2-fold by IMQ treatment in Traf6 $6^{f / f l}$ mice, and this IMQ-induced upregulation was attenuated in Traf6EKO mice (Figure 2, A and B). These genes included many psoriasis signature genes (15), including those coding defensins, S100a8/a9, and Ccl2 (Figure 2B and Supplemental Table 1). Pathway analysis revealed that various immune mediator-related pathways, including the TNF signaling pathway, which is a therapeutic target in psoriasis, were significantly upregulated by IMQ treatment in Traf6 $6^{\text {flfl }}$ mice compared with Traf6EKO mice (Figure 2C and Supplemental Table 2). Differences in the gene expression levels between the untreated Traf6 ${ }^{\sharp l / f l}$ mice and TrafbEKO mice were not annotated to specific pathways. These findings suggest that the IL-17-mediated psoriatic immune response induced by IMQ treatment is dependent on TRAF6 in keratinocytes.

To assess whether the IL-17-related immune response is impaired in Traf6EKO mice, we examined the expression of Il23a and Il12b encoding IL-23p19 and p40, respectively, and Th17 cytokines Il17a, Ill7f, and Il22, in the IMQ-treated skin by quantitative reverse transcription PCR (RT-PCR). During IMQ-induced psoriatic dermatitis, IL-23 is produced by DCs to induce an IL-17-mediated immune response $(32,33)$. Indeed, expression levels of the IL-23 subunit gene transcripts were upregulated in the skin of Traf6 $6^{f / f l}$ mice by treatment with IMQ (Figure 2D). Similarly, expression of $I l 17 a, I l 17 f$, and $I l 22$ were significantly upregulated in Traf6 $6^{\sharp l / l}$ mice by IMQ treatment (Figure 2D). The expression of other psoriasis-related genes, such as Saa1, Illa $I l 1 b$, Il24, and S100a8, was also induced in Traf6 $6^{A l f l}$ mice (Figure 2D and Supplemental Figure 2). In contrast, the IMQ-induced gene expression of all of these cytokines and chemokines was defective in Traf6EKO mice (Figure 2D and Supplemental Figure 2).

We next asked whether Traf6EKO mice are selectively deficient in the induction by IMQ of an IL-17related immune response or are broadly deficient in other cutaneous immune responses. To this end, we evaluated the requirement for keratinocyte TRAF6 in 2 distinct immune responses elucidated by epicutaneous treatments: treatment with 2,4-dinitro-1-fluorobenzene (DNFB) induces a Th1-type contact hypersensitivity, while repetitive papain occlusive dressing treatment induces a Th2 immune response $(34,35)$. DNFB treatment after sensitization elicited contact hypersensitivity with similar ear swelling in both Traf6EKO and Traf6 ${ }^{\theta / f l}$ mice (Supplemental Figure 3A). Although a significant but partial attenuation of Ifng expression was observed in the skin of Traf6EKO mice compared with that of Traf6 $6^{l / f l}$ mice, the induction of Ill7a gene expression was comparable in both groups (Supplemental Figure 3, B and C). In papain-induced skin inflammation, expression of $I l 4 \mathrm{mRNA}$ and serum IgE levels were comparable between Traf6EKO and Traf6 ${ }^{l / f l}$ mice (Supplemental Figure 3, D-F). Taken together, these findings indicate that TRAF6-dependent signaling in murine keratinocytes plays a critical role in the development of IL-17-related inflammation induced by IMQ treatment, but not in hapten-induced Th1-type inflammation or papain-induced Th2-type inflammation.

TRAF6 in keratinocytes is required for the activation of skin-resident DCs. Skin-resident DCs are reported to be the main source of IL-23 in the IMQ animal model $(31,33)$ and we, therefore, expected that the attenuated expression of IL-23 in the lesional skin of Traf6EKO mice (Figure 2D) was due to the impaired DC activation. We examined DC activation markers in both Traf6 $6^{f / f l}$ and TrafbEKO mice treated with IMQ. Skin-resident 
A

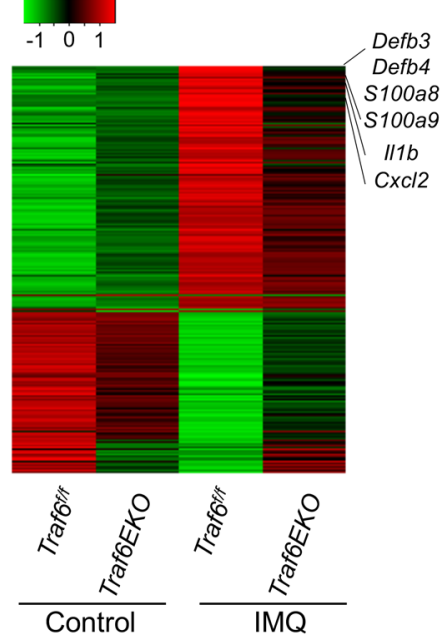

B

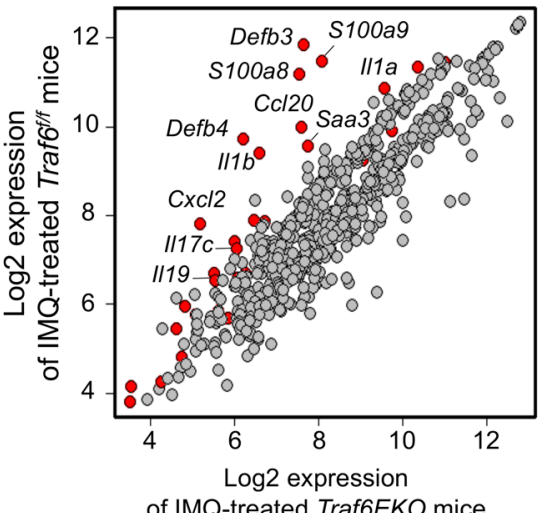

of IMQ-treated Traf6EKO mice

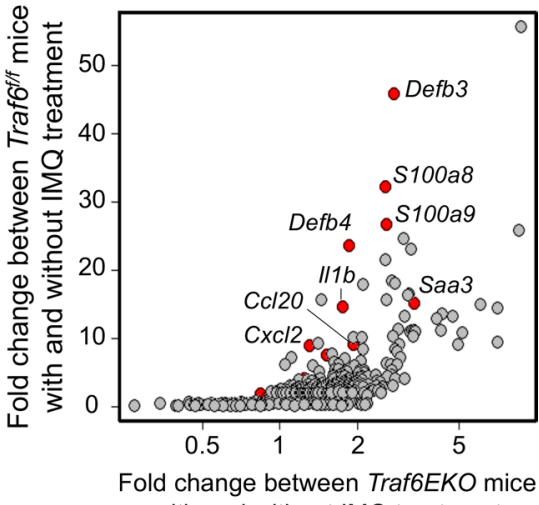

with and without IMQ treatment

C

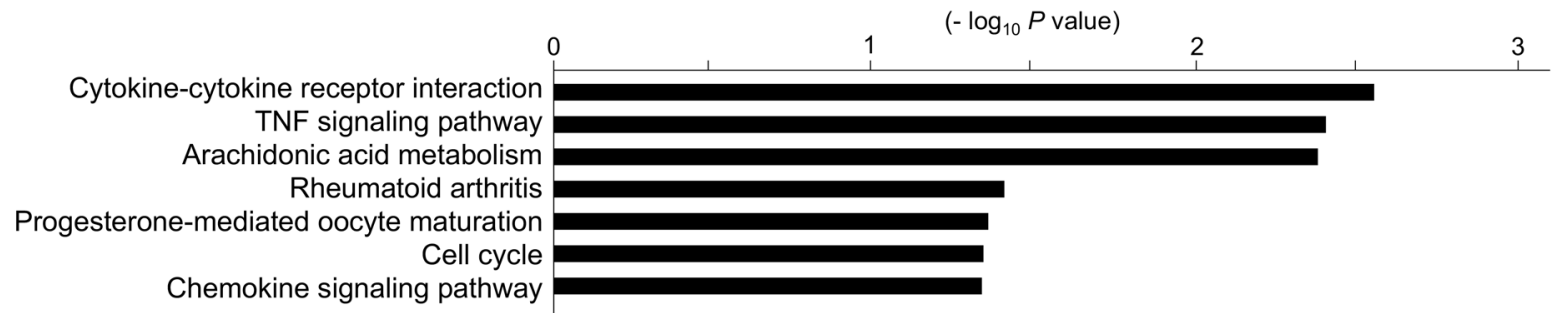

D

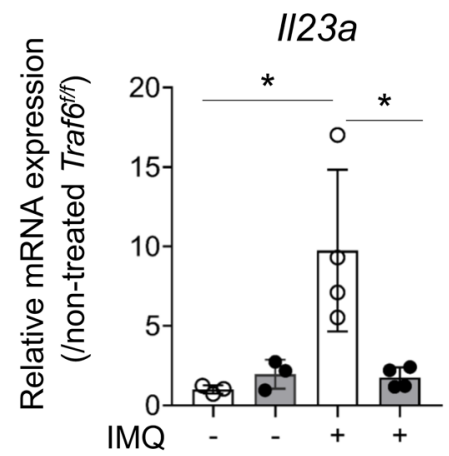

$\| 12 b$

$1 / 17 a$

$\| 17 f$
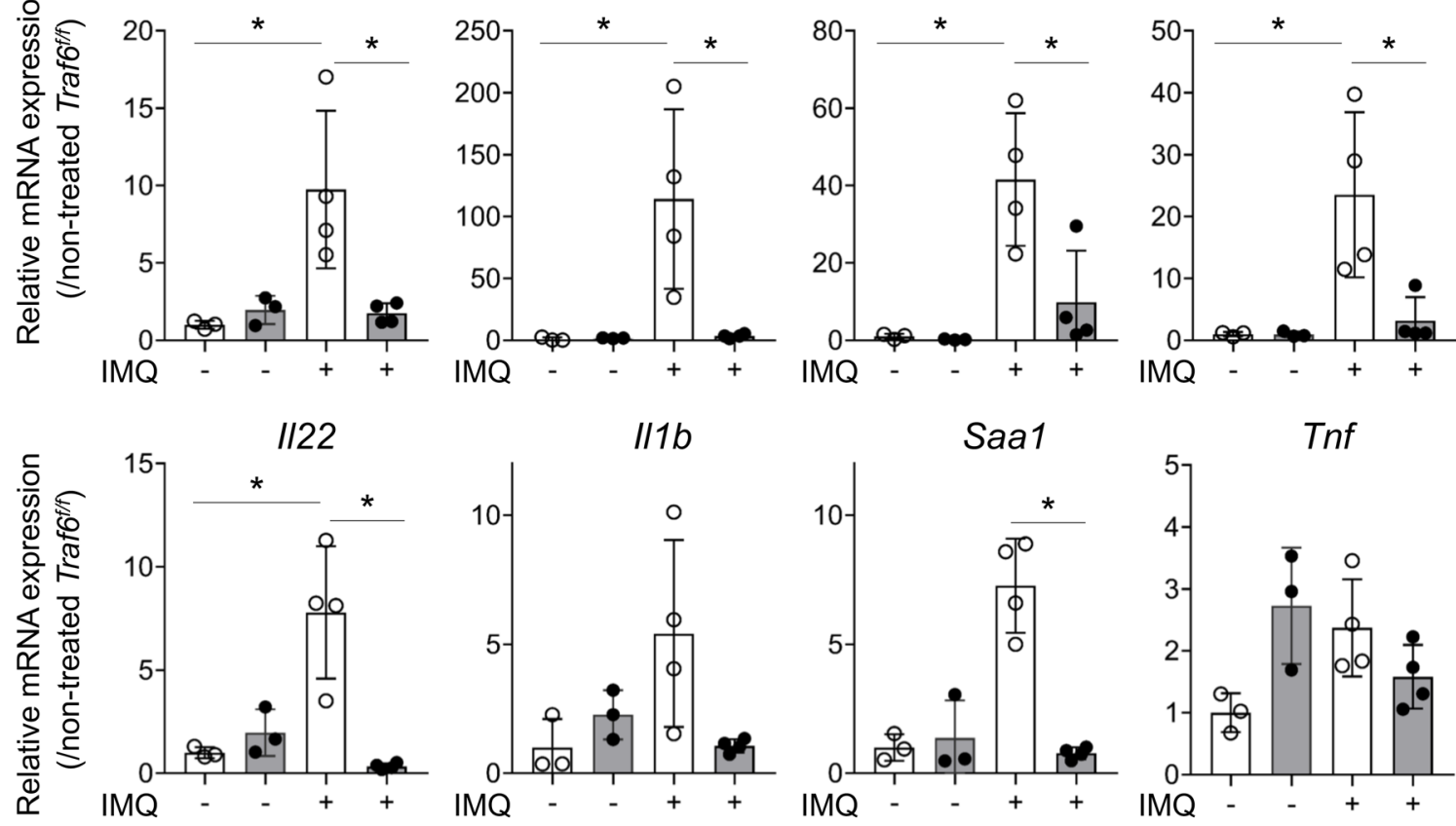

Traf6fff

Traf6EKO

Figure 2. IL-17-mediated gene expression was impaired in Traf6EKO mice. (A) Heatmap of differentially expressed genes in Traf6EKO mice and Traf6f/fl mice with or without IMQ treatment. Genes differentially expressed more than 2-fold by IMQ treatment in either mouse group (674 genes) were analyzed. Representative psoriasis-related genes are indicated. (B) Scatter plots of gene expression in Traf6EKO mice and Traf6fl/f mice. Left panel shows correlation of gene expression between IMQ-treated Traf6EKO mice and IMQ-treated Traf6 ${ }^{f / f l}$ mice. Right panel shows correlation between fold changes in gene expression induced by IMQ treatment in Traf6EKO mice and in Traf6 $6^{f / f}$ mice. Representative psoriasis-related genes are indicated by red plots accompanied with their names. (C) Pathways enriched in genes upregulated by IMQ treatment in Traff $f^{f / f}$ mice compared with Traf6EKO mice. Pathways with $P<0.05$ are shown. (D) qPCR analysis of mRNA levels in the epidermis of the IMQ-treated mouse ears on day $2(n \geq 3)$. The results were normalized to Gapdh expression and are shown as means $\pm \mathrm{SD}$. ${ }^{*} P<0.05$ (Tukey's multiple-comparisons test). Data are representative of 3 experiments (D) or 1 experiment $(\mathbf{A}-\mathbf{C}$ ). 
A

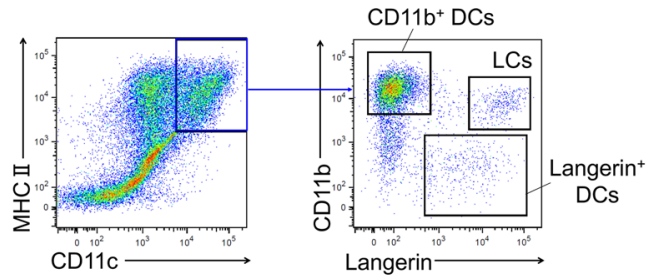

B
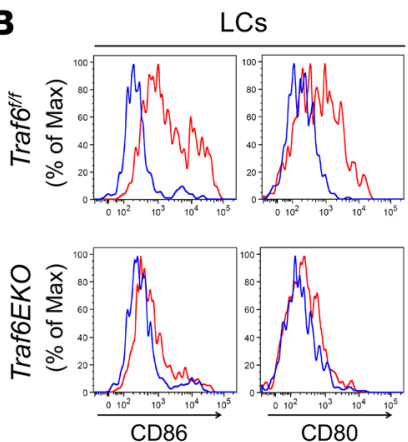

$\mathrm{CD} 11 \mathrm{~b}^{+} \mathrm{DCs}$
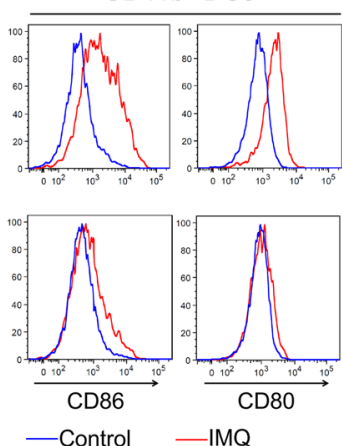

Langerin ${ }^{+}$DCs
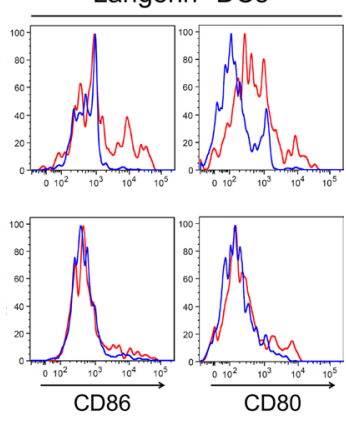

Figure 3. TRAF6 in keratinocytes is required for the activation of skin-resident DCs. (A) Flow cytometric analyses of skin-resident DCs. Gating strategies of Langerhans cells, langerin ${ }^{+} \mathrm{DCs}$, and CD11b+ DCs in the IMQ-treated ears are shown.

(B) Expression of activation markers in cutaneous DCs after IMQ treatment. Expression of CD86 and CD80 in cutaneous DCs was analyzed using the IMQ-treated ears from Traf6EKO mice and Traf6 ${ }^{f / f l}$ mice harvested on day $2(n=3)$. Representative single-parameter histograms of each DC subset in the ear treated with or without IMQ are shown in a red and blue line, respectively. (C) Time course of the rates of $\mathrm{CD}^{2} 6^{+}$and CD80 ${ }^{+}$populations in each DC subset after IMQ treatment $(n=3)$. Results are shown as means \pm SD. ${ }^{*} P<0.05$ (2-tailed Student's $t$ test). Data are representative of 2 experiments $(\mathbf{A}-\mathbf{C})$.

\section{C}
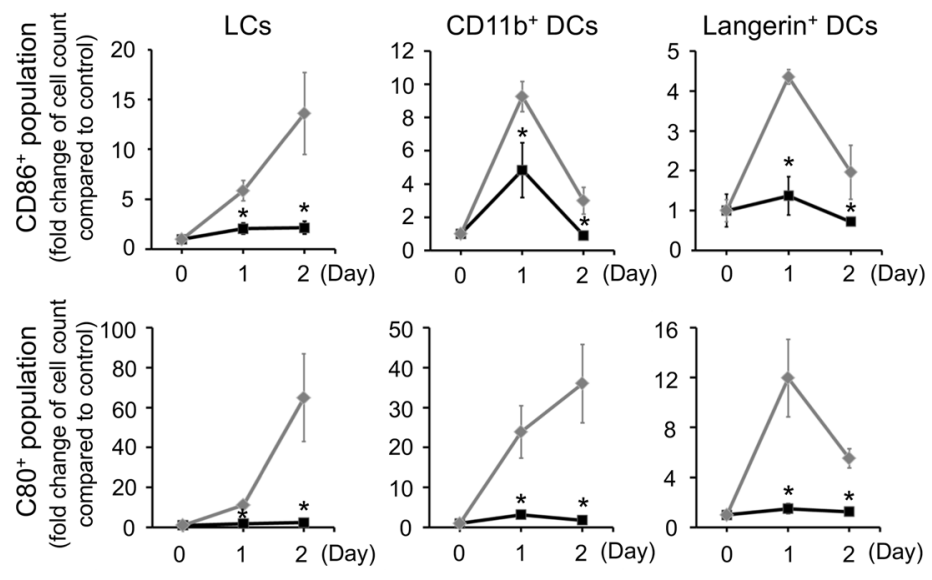

$\multimap \operatorname{Traf}^{\prime / f}$

DCs including LCs, langerin ${ }^{+}$DCs, and CD11b+ DCs (Figure 3A), exhibited high expression of CD80 and CD86 at the early ( $\leq 2$ days) after IMQ treatment in Traf6 $6^{A / f}$ mice, but not in Traf6EKO mice (Figure 3, B and C). These results suggested that TRAF6-dependent keratinocyte signaling is required for the activation of DCs in vivo. Previous studies suggested that IMQ directly activates skin-resident DCs and that this activation of DCs is associated with IL-23 production and subsequent IL-17 responses $(17,33)$. However, taken together with the marked decrease in the number of activated DCs and IL-17-producing $\gamma \delta$ T cells in the lesional skin of Traf6EKO mice (Figure 1, D and F), our results suggest that TRAF6-dependent keratinocyte signaling events are required for the skin-resident DC activation and induction of IL-17-producing $\gamma \delta$ T cells in IMQinduced inflammation, independent of the ability of IMQ to directly activate resident DCs.

Keratinocytes produce psoriasis-related mediators via TRAF6-dependent signaling in vitro. The results of cell death assays demonstrated that the susceptibility of keratinocytes to cell death did not depend on TRAF6 and that altered keratinocyte survival could not account for the defective inflammatory response of Traf6EKO mice (Supplemental Figure 4, A and B). We therefore hypothesized that loss of TRAF6 directly impairs signaling leading to transcription of psoriasis-related genes in keratinocytes, and that this loss of keratinocyte transcriptional responses abrogates development of IL-17-mediated inflammation. To confirm the function of TRAF6 in murine keratinocytes, we first evaluated IL-1 $\beta$ signaling in these cells. Consistent with observations made in the human keratinocyte cell line

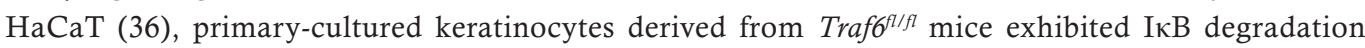




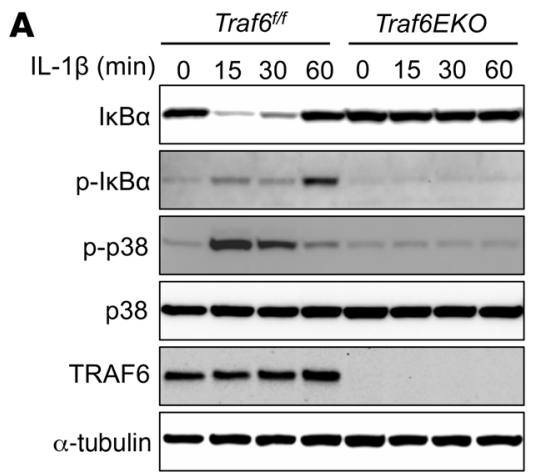

B
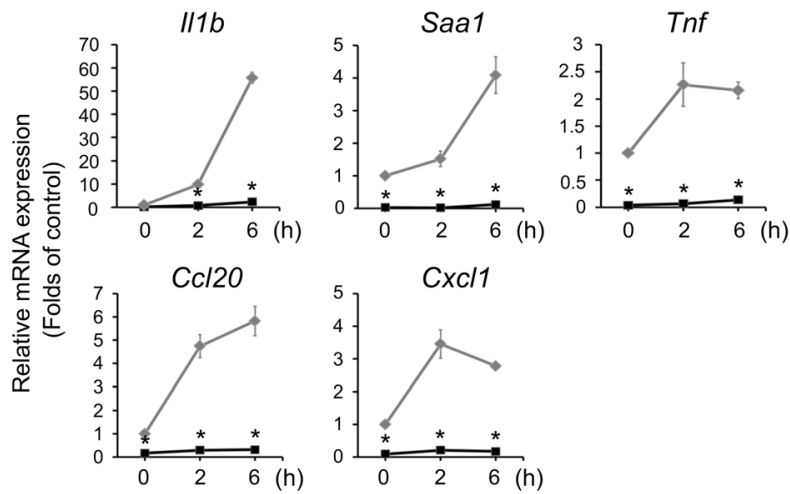

IMQ treatment

\section{C}

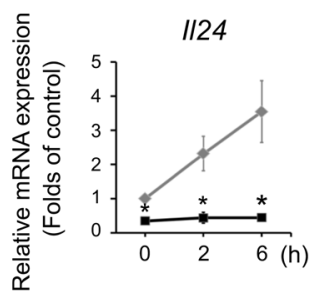

D

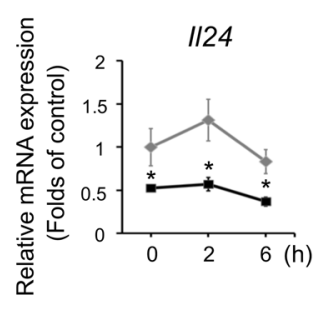

Cc/20

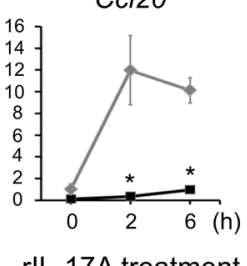

rlL-17A treatment

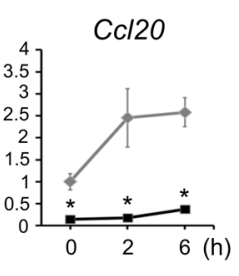

rIL-17F treatment

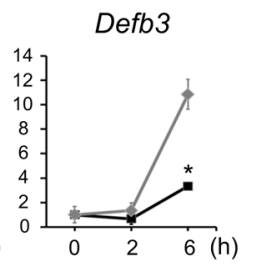

$\prec \operatorname{Traf6}^{t \prime \prime}$

$\rightarrow$ Traf6EKO
Figure 4. IMQ enhanced psoriasis-related gene expression in keratinocytes via TRAF6-dependent signaling pathway. (A) Western blot analysis of keratinocytes from Traf6EKO mice and Traf6 $\sigma^{\text {fl/fl }}$ mice stimulated with rlL-1 $\beta$ at the indicated time points. Total cell lysates were resolved by SDS-PACE followed by immunoblotting. (B) qPCR analysis of mRNA levels in the primary cultured keratinocytes from Traf6EKO mice and Traf6 $6^{f / / 7}$ mice stimulated with IMQ for 0-6 hours ( $n$ $=3$ ). (C and $\mathbf{D})$ qPCR analysis of mRNA levels in the primary cultured keratinocytes from Traf6EKO mice and Traf6 $f^{f / f}$ mice treated with IL-17A (100 ng/ml) (C) and IL-17F (100 ng/ $\mathrm{ml})(\mathbf{D})$ for $0-6$ hours $(n=3)$. These results were normalized to Gapdh expression and are shown as means \pm SD. ${ }^{*} P<$ 0.05 (2-tailed Student's $t$ test). Data are representative of 3 experiments (B) or 2 experiments (A, C, and $\mathbf{D})$.

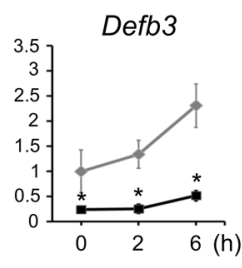

$\rightarrow-\operatorname{Traf} f^{t / 4}$

$\rightarrow$ Traf6EKO

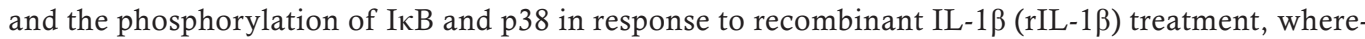
as those signaling events were not detected in TRAF6-deficient keratinocytes (Figure 4A).

We next investigated whether TRAF6 in keratinocytes is also required for the direct transcriptional activation by IMQ stimulation in vitro. Consistent with in vivo results (Figure 2D and Supplemental Figure 2), IMQ-treated Traf6 $6^{f l f l}$ keratinocytes showed significant upregulation of the expression levels of psoriasisrelated genes, such as Il1b, Saa1, Tnf, Ccl20, and Cxcl1 in vitro. However, the induction of all these genes was abrogated by the lack of TRAF6 (Figure 4B), despite intact phosphorylation of p38 without detectable activation of NF- $\mathrm{NB}$ in both groups (Supplemental Figure 5).

TRAF6 is required for the IL-17R signaling pathway in mouse embryonic fibroblasts (37). Therefore, we examined the potential role of epithelial TRAF6 in the IL-17R signaling pathway in keratinocytes. As expected, Traf6 $6^{\text {flfl}}$ keratinocytes produced Il24, Ccl20, and Defb3 by stimulation with IL-17A or IL-17F in vitro, whereas the expression of these genes in Traf6EKO keratinocytes was significantly 
impaired (Figure 4, C and D). Taken together, these results suggest that direct activation of epithelial cells by IMQ may be necessary for IL-17-mediated inflammation, and that TRAF6 is required for the transcriptional response of keratinocytes: both the primary response to external stimuli, such as IMQ, and the secondary response to IL-17.

IL-23 administration reconstitutes IL-17 production in Traf6EKO mice. The development of IMQ-induced psoriatic dermatitis and the expression of IL-17 is almost completely blocked in mice deficient for Il23a (16). Therefore, the reduced keratinocyte transcriptional responses accompanied by impaired activation of DCs and IL- $17^{+} \gamma \delta$ T cells in Traf6EKO mice could be attributable to decreased IL-23 production. This further suggests that induction of IL-23 production by skin DCs and other immune cells depends on the activation of TRAF6 signaling in keratinocytes in this animal model. However, the defect we observed in the responses of TRAF6-deficient keratinocytes to IL-17 in vitro suggests there may be an additional function for TRAF6 downstream of IL-23 production. To directly test whether the requirement for TRAF6dependent signaling in keratinocytes is predominantly for the induction of IL-23 production, Traf6 ${ }^{f / f t}$ and Traf6EKO mice were subcutaneously injected with rIL-23, which induces psoriatic dermatitis by directly inducing dermal $\gamma \delta$ T cells to produce IL-17 and IL-22 (38). Consistent with previous reports, Traff $f^{l / f l}$ mice developed psoriasis-like disease, as determined by ear thickness and histopathology (Figure 5, A and B). In contrast, IL-23 injection into Traf6EKO mice induced a more limited psoriatic inflammation (Figure 5, A and B), despite inducing comparable levels of expression of genes encoding IL-17A/F and IL-22, mainly produced by $\gamma \delta$ T cells (38), in both groups (Figure 5, C and D). These results demonstrated that the T cell repertoire in the skin of Traf6EKO mice was fully competent to mediate an IL-17 response. Furthermore, given that IL-17 production is fully reconstituted by IL-23 administration, these results suggest that IL-23 is a major downstream effector of the TRAF6-dependent keratinocyte response to external stimuli.

The unexpectedly less severe phenotype in the IL-23-treated Traf6EKO mice suggested that further activation of the IL-17R signaling, which is required for psoriatic inflammation following IL-23 production (38), depends on TRAF6 in keratinocytes. In fact, the increase in expression of genes mainly transcribed in keratinocytes, such as $C c l 20$ and Defb3, was significantly impaired in TrafbEKO mice (Figure 5C). This blunted response is potentially explained by defective IL-17R signaling in Traf6EKO keratinocytes (Figure 4, C and D). Moreover, the impaired expression of both Il24 and Il19 in Traf6EKO (Figure 5C) mice might also be responsible for the impaired phenotype, because of the essential role of these cytokines in IL-23induced inflammation (39).

Taken together, these results demonstrated that TRAF6 in keratinocytes plays an important role in the full development of psoriatic skin inflammation. Keratinocyte TRAF6 is a necessary primary, triggering event for the induction of the IL-23/IL-17 axis and for induction of fulminant psoriatic dermatitis as a key mediator of IL-17-driven keratinocyte-positive feedback pathways.

\section{Discussion}

In the present study, we demonstrated that mice lacking TRAF6 specifically in keratinocytes do not develop psoriatic inflammation in response to topical treatment with IMQ. Consistent with the known role of TRAF6 in TLR signaling (26) and IL-17R signaling (11, 39), we find that IMQ or IL-17 directly induced the expression of psoriasis-related genes in keratinocytes in a TRAF6-dependent manner. However, we also found that TRAF6-dependent keratinocyte responses were crucial for the activation of DCs, IL-17-producing $\gamma \delta \mathrm{T}$ cells, and the initiation of IL-17-mediated immunity. In addition, even when the primary responses of keratinocytes or DCs to IMQ were bypassed by direct administration of IL-23, development of psoriatic dermatitis was blunted in the absence of keratinocyte TRAF6. This reduction in IL-23/IL-17-driven psoriatic pathology likely reflects the role of TRAF6 in regulating epidermal IL-17R signaling. These findings indicate that TRAF6-dependent signaling in keratinocytes plays critical roles in the initiation as well as the propagation of IL-17-mediated immunity.

Given that epithelial cells are directly exposed to the external environment, it is reasonable to consider that upon external stresses, epithelial cells might trigger immune cell activation (15). Keratinocytes' contributions to the onset of psoriasis have been proposed to occur via cell death-dependent mechanisms (40); nucleic acid and antimicrobial peptide LL-37/RNA complexes released by injured keratinocytes have been shown to activate DCs or surrounding keratinocytes resulting in IL-23 production $(15,41,42)$. However, our in vitro studies showed that TRAF6 does not affect IMQ-induced cell death (Supplemental Figure 4, A and B), but is nevertheless required for induction of DC activation and IL-23 production. Of note, our results suggest that 
A

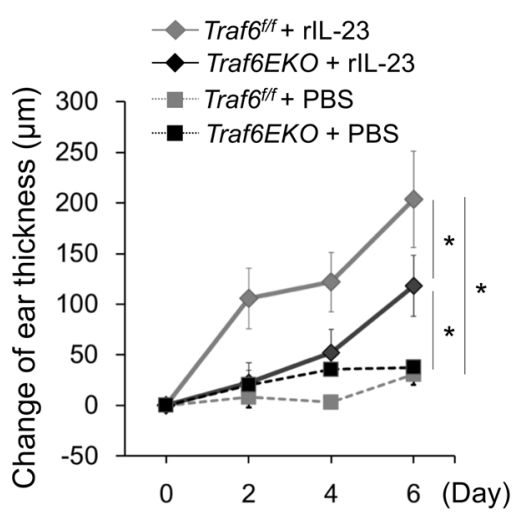

B

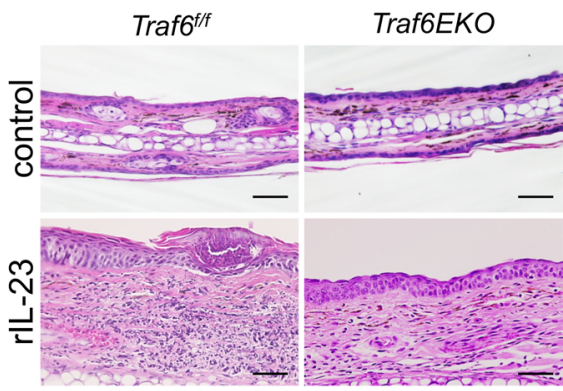

D

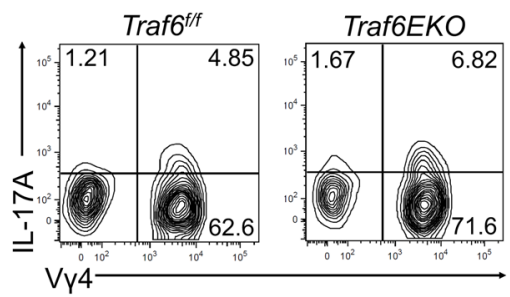

C
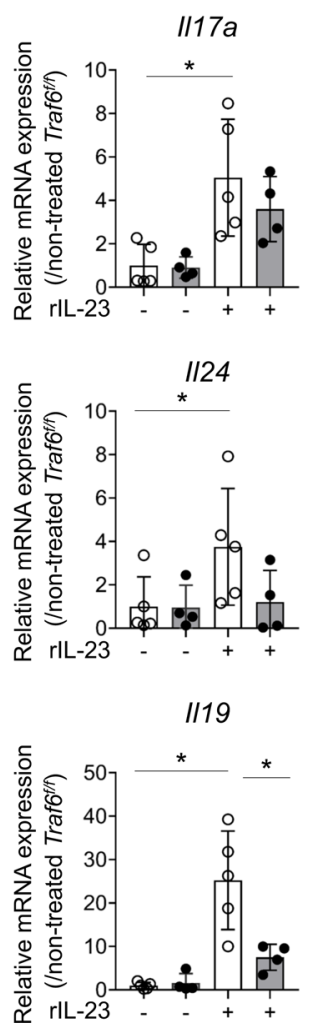
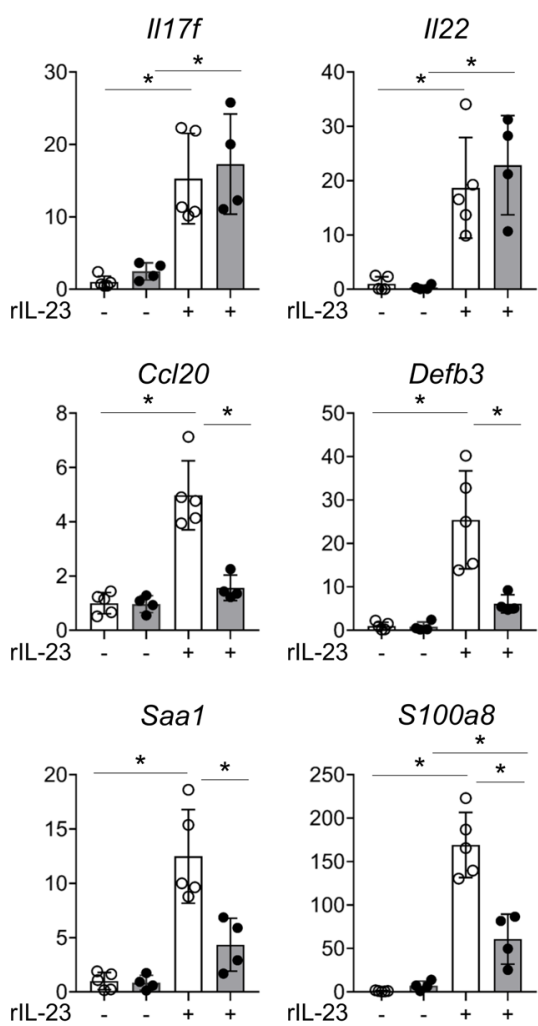

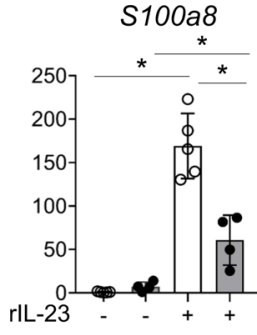

Figure 5. IL-23 partially restored the inflammation in Traf6EKO mice. (A) Time course of changes in ear thickness. TrafG $6^{f / f l}$ and Traf6EKO mice $(n \geq 4)$ were treated with rIL-23 every other day for 6 days and the ear thickness was measured before every treatment. Values are shown as means $\pm \mathrm{SD} .{ }^{*} P<0.05$ (2-tailed Student's $t$ test). (B) Histology of ear sections from the rIL-23-treated mice on day 6. H\&E staining. Scale bars: $50 \mu \mathrm{m}$. (C) qPCR analysis of mRNA levels in the ears from each group of mice on day $6(n \geq 4)$. The results were normalized to Gapdh expression and are shown as means \pm SD. ${ }^{*} P<0.05$ (Tukey's multiple-comparisons test). (D) Representative flow cytometry plots of IL-17A-producing $V_{\gamma} 4^{+} \gamma \delta$ T cells in the ears of Traf $\sigma^{f / f l}$ and Traf6EKO mice after 4 days of rlL-23 treatment. The plots show $\gamma \delta \mathrm{TCR}^{+} \mathrm{V} \gamma 4^{+} \mathrm{IL}-17 \mathrm{~A}^{+}$cells gated on $\mathrm{CD} 45^{+}$cells. Data are representative of 3 experiments ( $\mathbf{A}$ and $\mathbf{B}$ ) or $\mathbf{2}$ experiments (C and D).

TRAF6 in keratinocytes regulates the transcriptional expression of proinflammatory genes in the unstimulated state (Figure 4, B-D), and that epidermal keratinocytes might affect the local, steady-state cutaneous immune system even under nonpathological conditions. Thus, our work inspires questions and follow-up investigations about whether epithelial TRAF6 signaling is also involved in the physiological and pathological responses, such as to commensal microorganisms (e.g., candida species and Staphylococcus aureus) (13).

The pathogenic mechanisms by which immune cells are initially activated in psoriasis are still under debate (15). We found that TRAF6 in LCs was dispensable for IMQ-induced inflammation (Supplemental Figure 1C), consistent with results obtained when LCs were deleted (43). Our work does not contradict the possibility of the direct activation of immune cells by external stimuli in psoriatic inflammation. Nevertheless, we demonstrated that the primary response of keratinocytes is required to activate immune cells in IMQ-induced inflammation. However, the key TRAF6-dependent products from keratinocytes have not 
been identified. It has been demonstrated that IL-1R signaling also plays an important role in the development of psoriatic inflammation (44). We demonstrated that both the expression of IL-1 cytokines and the IL-1R signaling pathway depend on TRAF6 in keratinocytes (Figure 2, A, B and D; Figure 4A; and Supplemental Figure 2). These results may explain, in part, the impaired activation of skin immune cells and the abolishment of psoriatic inflammation in Traf6EKO mice.

In human psoriasis, the upstream signaling events that lead to TRAF6 activation in keratinocytes remain controversial. Although IMQ is a ligand for TLR7/8, several studies have demonstrated that IMQ induces TLR7/MyD88-independent signaling in keratinocytes $(45,46)$, and in vivo (47). Thus, it is conceivable that TLR7-independent TRAF6 signaling in keratinocytes would take part in the initial wave of psoriatic inflammation. CARD14, predominantly localized in the epidermis, is one of the candidate mediators of the TLR7-independent keratinocyte signaling pathways in psoriatic inflammation. Gain-of-function mutations in CARD14 induce the formation of signalosomes that activate TRAF6 $(24,48,49)$, and these mutations are found in familial-type psoriasis (50). Moreover, A20 encoded by another psoriasis susceptibility gene, Tnfaip3, also regulates the TRAF6 pathway (51). Therefore, these genetic susceptibilities may be in part related to the homeostatic imbalance in the epidermal TRAF6 signaling. After all, abolishment of psoriatic inflammation in Traf6EKO mice strongly suggests that the pathogenic signals in keratinocytes require TRAF6 regardless of their upstream signaling pathways.

Previous studies have suggested contributions of epithelial cells to immune divergence in several types of inflammation. However, to what extent, and by what principles epithelial cells are involved in shaping each response remains largely unknown. Here, we demonstrated that epithelial TRAF6 signaling is hardly involved in Th1 or Th2 responses: epicutaneous hapten-induced contact hypersensitivity or papain-induced dermatitis, respectively. Considering the requirement for epithelial TRAF6 for IMQ-induced IL-17-mediated inflammation, our results suggest a selective role for this specific epithelial signaling pathway. It is of great interest whether the TRAF6-independent epithelial signaling events are analogously restricted to induction of Th2 responses. Elucidation of such a pathway would be of particular interest given the protective role of the Th2 system in responses to helminths, arthropods, and toxins and the established role of keratinocytes in cutaneous Th2 response $(7,8)$.

It is noteworthy that studies using mice lacking TRAF6 in intestinal epithelial cells showed a critical role for intestinal epithelial TRAF6 for preventing propagation of dextran sodium sulfate-induced (DSSinduced) colon inflammation (52). Intestinal barrier integrity is maintained by IL-17 cytokines $(53,54)$. Thus, it is a plausible idea that epithelial TRAF6 is uniquely involved in local, IL-17-mediated, protective responses despite its pathogenetic role in IL-17-mediated psoriatic inflammation.

There are a few limitations to the current study. First, we could not fully rule out indirect effects of defective epithelial development or changes in the organization of immune cells in Traf6EKO mice. TRAF6 is involved in the development of epithelial appendages $(55,56)$, although we did not observe marked defects in epidermal development or keratinocyte proliferation. Second, we could not detect direct evidence of elevated TRAF6 E3 ubiquitin ligase activity in psoriasis, by the experiments with existing techniques or by analyzing the published human transcriptome.

In summary, we demonstrated that epithelial TRAF6-dependent signaling is essential for the initiation of IL-17-mediated inflammation induced by IMQ treatment in mice. In this animal model, epithelial cells respond to the extrinsic agent in a TRAF6-dependent manner, engaging a transcriptional program that programs resident immune cells, leading to the induction and promotion of cutaneous psoriatic inflammation. These findings raise further questions about whether epithelial cells sense microbes directly and how they orchestrate immune cells to induce the appropriate immune response, in the gut, in the lung, and in the skin. This work also underscores the importance of considering epithelial signaling pathways in efforts to identify new treatments for chronic inflammatory diseases and develop effective vaccination strategies for infectious agents.

\section{Methods}

Mice. Eight- to 12-week-old female mice were used in this study. All animals used were of the C57BL/6 genetic background. Traff ${ }^{l / f l}$ mice have been reported previously (18). To prepare keratinocyte-specific TRAF6-deficient (Traf6EKO) mice, male mice bearing the Cre recombinase transgene, under the control of the keratin 5 promoter (K5-Cre), were mated with female Traf6 $6^{f / f l}$ mice (18). The K5-Cre; Traf6 ${ }^{f / f l}$ mice were born at expected Mendelian frequency, fertile, and developed normally without gross abnormality, and displayed impaired expression of Traf6 mRNA (Supplemental Figure 1A) and TRAF6 protein in keratinocytes (Figure 4A). 
The male Langerin-Cre mice were crossed with the female Traff $f^{l / f l}$ mice to create Langerin-Cre; Traf6 $6^{f / f l}$ (Traf6LCKO) mice, in which deletion of Traf6 should be limited to epidermal LCs (57). All animals were maintained under specific pathogen-free conditions.

Skin inflammation models. For the IMQ-induced psoriasis model, a daily dose of $10 \mathrm{mg}$ of IMQ-containing cream, Aldara (Beselna Cream 5\%, Mochida Pharmaceuticals), was applied onto the mouse ear for 2-6 consecutive days. In this study, the abbreviation IMQ always means "IMQ-containing cream, Aldara" unless otherwise indicated. Ear thickness was measured for each mouse every day using a thickness gauge (Teclok), and the difference from day 0 was expressed as the change in ear thickness. For DNFB-induced contact hypersensitivity, mice were sensitized on shaved abdominal skin with $25 \mu 10.5 \%$ (w/v) DNFB in acetone/olive oil (4:1). Five days after sensitization, the ears were challenged with an application of $20 \mu 1$ $0.3 \%$ DNFB. For the papain occlusive dressing technique, $100 \mu \mathrm{g}$ of papain (Calbiochem) diluted in $10 \mu \mathrm{l}$ PBS was applied on shaved and tape-stripped dorsal skin and fixed. Each mouse had a total of three 4-day exposures to the patch, separated by 3-day intervals. Mice were euthanized at the end of the third cycle of sensitization. The clinical severity was scored according to the macroscopic diagnostic criteria (58). For the IL-23-induced psoriatic dermatitis model, mouse rIL-23 (500 ng in $20 \mu \mathrm{l}$ of PBS) (Wako Pure Chemicals) was subcutaneously injected into one ear of each mouse using a 30-gauge needle every other day for 6 days.

Histological analyses. For histological analyses, skin tissues were fixed with $10 \%$ formalin in PBS and then embedded in paraffin. Sections with a thickness of $5 \mu \mathrm{m}$ were prepared and stained with hematoxylin and eosin (H\&E).

Flow cytometry. Flow cytometric analysis was performed by the following methods. Briefly, a split ear sample was incubated for 60 minutes at $37^{\circ} \mathrm{C}$ with a solution of complete RPMI containing $100 \mu \mathrm{g} /$ $\mathrm{ml}$ DNase I (Sigma-Aldrich) and $2 \mathrm{mg} / \mathrm{ml}$ Liberase TL (Roche) to obtain a homogeneous cell suspension. The cell suspensions were filtered using a $40-\mu \mathrm{m}$ cell strainer and stained. For analysis of intracellular cytokine production, cell suspensions were obtained in the presence of $10 \mu \mathrm{g} / \mathrm{ml} \mathrm{brefeldin}$ A (Sigma-Aldrich) and were fixed with Cytofix Buffer and permeabilized with Perm/Wash Buffer according to the manufacturer's protocol (BD Biosciences). The following antibodies were used: antibody against mouse CD4-FITC (RM4-5), anti-CD8a-PerCP-Cy5.5 (53-6.7), anti-CD11c-BV605 (N418), anti-MHC class II-FITC (M5/114.15.2), anti-TCR- $\beta$-PE-Cy7 (H57-597), anti- $\gamma \delta$ TCR-FITC/PB (GL3), anti-V $\gamma 2-$ PE/APC (UC3-10A6), and anti-Ly6G-FITC (1A8) (all from BioLegend); anti-CD11b-PE-Cy7 (M1/70), anti-CD45-PE-Cy7/PB (30-F11), anti- $\gamma \delta$ TCR-FITC (GL3), and anti-IL-17A-PE (TC11-18H10) (all from BD Biosciences); anti-CD80-PE (16-10A1), anti-CD86-PE (GL1), and anti-langerin-APC (CD207) (RMUL.2) (all from eBioscience). Flow cytometry was done with an LSR Fortessa (BD Biosciences) and data were analyzed with FlowJo software (Tree Star).

$R N A$ isolation and quantitative RT-PCR analysis. Total RNA was isolated using TRIzol (Invitrogen) or RNeasy kits (Qiagen) according to the manufacturers' protocol. Complementary DNA was reverse transcribed using the Prime Script RT reagent kit (Takara Bio). Quantitative RT-PCR was performed as reported previously (34). All primers were obtained from Greiner Japan. Primer sequences are provided in Supplemental Table 3.

Microarray analysis. Total RNAs prepared from the ears of Traf6EKO mice and Traf6 ${ }^{f / f l}$ mice treated with IMQ for 2 consecutive days $(n=2)$ were analyzed by microarray. Total RNA from skin samples was immediately isolated with TRIzol according to the manufacturer's protocol. An amplified sense-strand DNA product was synthesized with the Ambion WT Expression Kit (Life Technologies), was fragmented and labeled by the WT Terminal Labelling and Controls Kit (Affymetrix), and hybridized to a Mouse Gene 1.0 ST Array (Affymetrix). We used the robust multiarray average algorithm for log transformation $\left(\log _{2}\right)$ and normalization of the GeneChip data. Z-score transformation followed by generation of a heatmap was performed using R software. Kyoto Encyclopedia of Genes and Genomes (KEGG) pathway enrichment analysis was performed using DAVID v6.8 bioinformatics resources (https://david.ncifcrf.gov/). $P$ values $<0.05$ were considered to be significant (adjusted by the Benjamini-Hochberg method), whereas false discovery rates of only the top 2 pathways were $<0.1$. Microarray data were deposited in the NCBI's Gene Expression Omnibus database (GEO GSE101077).

Cell culture. Mouse primary keratinocytes were harvested from newborn epidermis and were cultured in low-calcium medium $\left(0.05 \mathrm{mM} \mathrm{Ca}^{2+}\right)$, using EMEM (Lonza) with chelated FCS as reported previously (59). In general, full-thickness skin was obtained from newborn mice. The skin samples were floated and incubated in Dispase II ( $1 \mathrm{U} / \mathrm{ml}$; Roche) overnight at $4^{\circ} \mathrm{C}$. The epidermis was then separated from the 
dermis, cut into pieces, and incubated in $0.05 \%$ trypsin for 5 minutes. Cells were cultured in low-calcium medium $\left(0.05 \mathrm{mM} \mathrm{Ca}^{2+}\right)$, using EMEM with chelated FCS. Cells were seeded on 12-well plates at a density of $3 \times 10^{5}$ cells per well for RNA extraction and lactate dehydrogenase (LDH) assay. For cell-signaling experiments, cells were serum starved for 24 hours prior to stimulation. A total of $60 \mathrm{mg}$ IMQ (Aldara cream) was dissolved in $1 \mathrm{ml}$ dimethylsulfoxide (DMSO), which was further diluted with cell-culture medi$\mathrm{um}$ at the indicated dilution, and used for cell stimulation. IMQ (30 $\mu \mathrm{g} / \mathrm{ml})$, rIL-17A and IL-17F (100 ng/ $\mathrm{ml}$; R\&D Systems), and $\mathrm{rIL}-1 \beta$ ( $20 \mathrm{ng} / \mathrm{ml}, \mathrm{R} \& \mathrm{D}$ Systems) were used to stimulate primary keratinocytes. LDH assay was performed using a Pierce LDH cytotoxicity assay kit (Thermo Fisher Scientific) according to the manufacturer's protocol.

Cell death assay. We treated primary cultured murine keratinocytes with or without IMQ and analyzed the release of LDH in the supernatants. The levels of LDH release were comparable between Traff $6^{\prime l f l}$ and Traf6EKO mice at all tested doses of IMQ, and nuclear morphology was indistinguishable in both at the lower doses of IMQ (Supplemental Figure 4, A and B).

Western blotting. Cells were lysed with cell lysis buffer (Abcam, ab152163) and protease inhibitor cocktail (Sigma-Aldrich, P8340). The lysates were centrifuged at 13,000 $\mathrm{g}$ for 5 minutes at $4^{\circ} \mathrm{C}$ and the supernatant was used in the following steps. Samples were resolved by SDS-PAGE and transferred to nitrocellulose membranes (Bio-Rad, 162-0168). After blocking with 5\% (wt/vol) nonfat dry milk or bovine serum albumin, the membranes were incubated with primary antibodies for 16 hours at $4^{\circ} \mathrm{C}$, followed by incubation with horseradish peroxidase-conjugated secondary antibodies for 1 hour at room temperature. Signals were detected using ECL Prime Western Blotting Detection Reagent (GE Healthcare, GE-RPN2024). Images were captured and density of the bands was measured using the ChemiDoc Touch Imaging System (Bio-Rad).

Antibodies recognizing the following proteins were used: TRAF6 (Santa Cruz Biotechnology, 7221), $\alpha$-tubulin (Sigma-Aldrich, T5168), IкB $\alpha$ (Cell Signaling Technology, 9242), p-IкB $\alpha$ (Cell Signaling Technology, 2859), p38 (Cell Signaling Technology, 9212), and p-p38 (Cell Signaling Technology, 9211).

Statistics. Unless otherwise indicated, data are presented as means \pm standard deviations. Twotailed Student's $t$ test and Tukey's multiple comparison test were performed to assess statistical significance. $P$ values $<0.05$ were considered to be significant and are marked by an asterisk in the figures.

Study approval. In this study, all procedures for animal experiments were reviewed and approved by Animal Research Committee, Graduate School of Medicine, Kyoto University (approval Med Kyo 17282) and were performed in accordance with institutional guidelines.

\section{Author contributions}

RM and TD designed the studies. RM performed experiments and analyzed data. TD advised on execution of experiments, and interpretation of results. ST and YS performed microarray. KJI, MT, and TK provided animals and research ideas. TN generated animals. AK, TH, GE, AO, and SN reviewed the draft. MSH, $\mathrm{KS}$, and YN contributed to discussion and interpretation of the results. RM, TD and MSH wrote and edited the manuscript. KK supervised and financed the work.

\section{Acknowledgments}

We thank Shuh Narumiya, Eiryo Kawakami, and Tomonori Matsumoto for helpful comments. This work was supported by JSPS KAKENHI grant number $15 \mathrm{H} 05790$.

Address correspondence to: Teruki Dainichi or Kenji Kabashima, Department of Dermatology, Kyoto University Graduate School of Medicine, 54 Shogoin Kawahara-cho, Sakyo, Kyoto 606-8507, Japan. Phone: 81.75.751.3310; Email; dainichi@kuhp.kyoto-u.ac.jp (T. Dainichi); kaba@kuhp.kyoto-u.ac.jp (K. Kabashima).

1. Müller L, Jaspers I. Epithelial cells, the "switchboard" of respiratory immune defense responses: effects of air pollutants. Swiss Med Wkly. 2012;142:w13653.

2. Nowarski R, Jackson R, Flavell RA. The stromal intervention: Regulation of immunity and inflammation at the epithelialmesenchymal barrier. Cell. 2017;168(3):362-375.

3. Iwasaki A, Foxman EF, Molony RD. Early local immune defences in the respiratory tract. Nat Rev Immunol. 2017;17(1):7-20.

4. Leyva-Castillo JM, Hener P, Jiang H, Li M. TSLP produced by keratinocytes promotes allergen sensitization through skin and thereby triggers atopic march in mice. J Invest Dermatol. 2013;133(1):154-163. 
5. Ivanov II, et al. Induction of intestinal Th17 cells by segmented filamentous bacteria. Cell. 2009;139(3):485-498.

6 . Yoo J, et al. Spontaneous atopic dermatitis in mice expressing an inducible thymic stromal lymphopoietin transgene specifically in the skin. J Exp Med. 2005;202(4):541-549.

7. Li M, Hener P, Zhang Z, Kato S, Metzger D, Chambon P. Topical vitamin D3 and low-calcemic analogs induce thymic stromal lymphopoietin in mouse keratinocytes and trigger an atopic dermatitis. Proc Natl Acad Sci USA. 2006;103(31):11736-11741.

8. Li M, Hener P, Zhang Z, Ganti KP, Metzger D, Chambon P. Induction of thymic stromal lymphopoietin expression in keratinocytes is necessary for generating an atopic dermatitis upon application of the active vitamin D3 analogue MC903 on mouse skin. J Invest Dermatol. 2009;129(2):498-502.

9. Wolfram JA, et al. Keratinocyte but not endothelial cell-specific overexpression of Tie2 leads to the development of psoriasis. Am J Pathol. 2009;174(4):1443-1458.

10. Johnston A, et al. Keratinocyte overexpression of IL-17C promotes psoriasiform skin inflammation. J Immunol. 2013;190(5):2252-2262.

11. Ha HL, et al. IL-17 drives psoriatic inflammation via distinct, target cell-specific mechanisms. Proc Natl Acad Sci USA 2014;111(33):E3422-E3431

12. Ippagunta SK, et al. Keratinocytes contribute intrinsically to psoriasis upon loss of Tnip1 function. Proc Natl Acad Sci USA 2016;113(41):E6162-E6171.

13. Nakagawa S, et al. Staphylococcus aureus virulent PSM $\alpha$ peptides induce keratinocyte alarmin release to orchestrate IL-17-dependent skin inflammation. Cell Host Microbe. 2017;22(5):667-677.e5.

14. Nestle FO, Kaplan DH, Barker J. Psoriasis. N Engl J Med. 2009;361(5):496-509.

15. Lowes MA, Suárez-Fariñas M, Krueger JG. Immunology of psoriasis. Annu Rev Immunol. 2014;32:227-255

16. van der Fits L, et al. Imiquimod-induced psoriasis-like skin inflammation in mice is mediated via the IL-23/IL-17 axis. J Immunol. 2009;182(9):5836-5845.

17. Di Cesare A, Di Meglio P, Nestle FO. The IL-23/Th17 axis in the immunopathogenesis of psoriasis. J Invest Dermatol. 2009;129(6):1339-1350.

18. Kobayashi T, et al. TRAF6 is a critical factor for dendritic cell maturation and development. Immunity. 2003;19(3):353-363.

19. Yoon K, Jung EJ, Lee SR, Kim J, Choi Y, Lee SY. TRAF6 deficiency promotes TNF-induced cell death through inactivation of GSK3beta. Cell Death Differ. 2008;15(4):730-738.

20. Zenz R, et al. Psoriasis-like skin disease and arthritis caused by inducible epidermal deletion of Jun proteins. Nature. 2005;437(7057):369-375.

21. Kumari S, et al. Tumor necrosis factor receptor signaling in keratinocytes triggers interleukin-24-dependent psoriasis-like skin inflammation in mice. Immunity. 2013;39(5):899-911.

22. Grinberg-Bleyer Y, et al. Cutting edge: NF-kB p65 and c-Rel control epidermal development and immune homeostasis in the skin. J Immunol. 2015;194(6):2472-2476.

23. Jordan CT, et al. Rare and common variants in CARD14, encoding an epidermal regulator of NF-kappaB, in psoriasis. Am $J$ Hum Genet. 2012;90(5):796-808.

24. Afonina IS, et al. The paracaspase MALT1 mediates CARD14-induced signaling in keratinocytes. EMBO Rep. 2016;17(6):914-927.

25. Callahan JA, et al. Cutting edge: ABIN-1 protects against psoriasis by restricting MyD88 signals in dendritic cells. J Immunol. 2013;191(2):535-539.

26. Chung JY, Park YC, Ye H, Wu H. All TRAFs are not created equal: common and distinct molecular mechanisms of TRAFmediated signal transduction. J Cell Sci. 2002;115(Pt 4):679-688.

27. Sumida $\mathrm{H}$, et al. Interplay between CXCR2 and BLT1 facilitates neutrophil infiltration and resultant keratinocyte activation in a murine model of imiquimod-induced psoriasis. J Immunol. 2014;192(9):4361-4369.

28. Pantelyushin S, et al. Roryt ${ }^{+}$innate lymphocytes and $\gamma \delta \mathrm{T}$ cells initiate psoriasiform plaque formation in mice. J Clin Invest. 2012;122(6):2252-2256

29. Sano S, et al. Stat3 in thymic epithelial cells is essential for postnatal maintenance of thymic architecture and thymocyte survival. Immunity. 2001;15(2):261-273.

30. Bonito AJ, et al. Medullary thymic epithelial cell depletion leads to autoimmune hepatitis. J Clin Invest. 2013;123(8):3510-3524.

31. Yoshiki R, et al. IL-23 from Langerhans cells is required for the development of imiquimod-induced psoriasis-like dermatitis by induction of IL-17A-producing $\gamma \delta$ T cells. J Invest Dermatol. 2014;134(7):1912-1921.

32. Lee E, et al. Increased expression of interleukin 23 p19 and p40 in lesional skin of patients with psoriasis vulgaris. $J$ Exp Med. 2004;199(1):125-130.

33. Wohn C, et al. Langerin(neg) conventional dendritic cells produce IL-23 to drive psoriatic plaque formation in mice. Proc Nat Acad Sci USA. 2013;110(26):10723-10728.

34. Nakajima S, et al. Prostaglandin I2-IP signaling promotes Th1 differentiation in a mouse model of contact hypersensitivity. J Immunol. 2010;184(10):5595-5603.

35. Iida $\mathrm{H}$, et al. Epicutaneous administration of papain induces $\operatorname{IgE}$ and $\operatorname{IgG}$ responses in a cysteine protease activity-dependent manner. Allergol Int. 2014;63(2):219-226.

36. Strickson S, et al. Roles of the TRAF6 and Pellino E3 ligases in MyD88 and RANKL signaling. Proc Natl Acad Sci USA. 2017;114(17):E3481-E3489.

37. Qian Y, et al. The adaptor Act1 is required for interleukin 17-dependent signaling associated with autoimmune and inflammatory disease. Nat Immunol. 2007;8(3):247-256.

38. Cai Y, et al. Pivotal role of dermal IL-17-producing $\gamma \delta$ T cells in skin inflammation. Immunity. 2011;35(4):596-610.

39. Chan JR, et al. IL-23 stimulates epidermal hyperplasia via TNF and IL-20R2-dependent mechanisms with implications for psoriasis pathogenesis. J Exp Med. 2006;203(12):2577-2587.

40. Pasparakis M, Haase I, Nestle FO. Mechanisms regulating skin immunity and inflammation. Nat Rev Immunol. 2014;14(5):289-301.

41. Ganguly D, et al. Self-RNA-antimicrobial peptide complexes activate human dendritic cells through TLR7 and TLR8. J Exp Med. 2009;206(9):1983-1994. 
42. Zhang LJ, et al. Antimicrobial peptide LL37 and MAVS signaling drive interferon- $\beta$ production by epidermal keratinocytes during skin injury. Immunity. 2016;45(1):119-130.

43. Greter M, et al. Stroma-derived interleukin-34 controls the development and maintenance of Langerhans cells and the maintenance of microglia. Immunity. 2012;37(6):1050-1060.

44. Rabeony H, et al. IMQ-induced skin inflammation in mice is dependent on IL-1R1 and MyD88 signaling but independent of the NLRP3 inflammasome. Eur J Immunol. 2015;45(10):2847-2857.

45. Schön MP, Schön M, Klotz KN. The small antitumoral immune response modifier imiquimod interacts with adenosine receptor signaling in a TLR7- and TLR8-independent fashion. J Invest Dermatol. 2006;126(6):1338-1347.

46. Drobits B, et al. Imiquimod clears tumors in mice independent of adaptive immunity by converting pDCs into tumor-killing effector cells. J Clin Invest. 2012;122(2):575-585.

47. Walter A, et al. Aldara activates TLR7-independent immune defence. Nat Commun. 2013;4:1560.

48. Scudiero I, Zotti T, Ferravante A, Vessichelli M, Vito P, Stilo R. Alternative splicing of CARMA2/CARD14 transcripts gener-

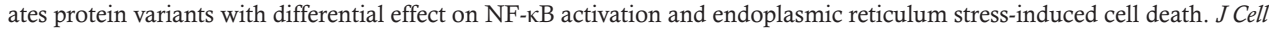
Physiol. 2011;226(12):3121-3131

49. Howes A, et al. Psoriasis mutations disrupt CARD14 autoinhibition promoting BCL10-MALT1-dependent NF-kB activation. Biochem J. 2016;473(12):1759-1768.

50. Jordan CT, et al. PSORS2 is due to mutations in CARD14. Am J Hum Genet. 2012;90(5):784-795.

51. Zhang Q, Lenardo MJ, Baltimore D. 30 years of NF-кB: A blossoming of relevance to human pathobiology. Cell. 2017;168(1-2):37-57.

52. Vlantis K, Polykratis A, Welz PS, van Loo G, Pasparakis M, Wullaert A. TLR-independent anti-inflammatory function of intestinal epithelial TRAF6 signalling prevents DSS-induced colitis in mice. Gut. 2016;65(6):935-943.

53. Pappu R, Rutz S, Ouyang W. Regulation of epithelial immunity by IL-17 family cytokines. Trends Immunol. 2012;33(7):343-349

54. Ogawa A, Andoh A, Araki Y, Bamba T, Fujiyama Y. Neutralization of interleukin-17 aggravates dextran sulfate sodiuminduced colitis in mice. Clin Immunol. 2004;110(1):55-62.

55. Naito A, et al. TRAF6-deficient mice display hypohidrotic ectodermal dysplasia. Proc Natl Acad Sci USA. 2002;99(13):8766-8771.

56. Kanaya T, et al. Development of intestinal $\mathrm{M}$ cells and follicle-associated epithelium is regulated by TRAF6-mediated NF- $\mathrm{kB}$ signaling. J Exp Med. 2018;215(2):501-519.

57. Kaplan DH, Li MO, Jenison MC, Shlomchik WD, Flavell RA, Shlomchik MJ. Autocrine/paracrine TGFbeta1 is required for the development of epidermal Langerhans cells. J Exp Med. 2007;204(11):2545-2552.

58. Leung DY, et al. Thymopentin therapy reduces the clinical severity of atopic dermatitis. J Allergy Clin Immunol. 1990;85(5):927-933.

59. Lichti U, Anders J, Yuspa SH. Isolation and short-term culture of primary keratinocytes, hair follicle populations and dermal cells from newborn mice and keratinocytes from adult mice for in vitro analysis and for grafting to immunodeficient mice. Nat Protoc. 2008;3(5):799-810. 